\title{
Alteración del desarrollo psicomotor en hijos de mujeres con depresión posparto de la ciudad de Valdivia-Chile
}

'Instituto de Pediatría, Facultad de Medicina, Universidad Austral de Chile, Valdivia. Chile.

${ }^{2}$ Departamento. Salud Pública-CIGES, Facultad de Medicina, Universidad de la Frontera, Temuco. Chile. ${ }^{3}$ Instituto de Enfermería, Facultad de Medicina, Universidad Austral, Valdivia. Chile.

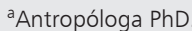

bioestadístico PhD.

'Enfermero. MA.

¿Epidemiología Clínica.

Fuente de Financiamiento: Proyecto FONIS SA0512011.

Recibido el 13 de diciembre de 2011, aceptado el 27 de noviembre de 2012

Correspondencia a: Ana M. Alarcón

Manuel Montt 112 Tel: (45)325740

E-mail: amalarc@ufro.cl

\author{
LORETO PODESTÁ L. ${ }^{1}$, ANA MARÍA ALARCÓN ${ }^{2, a}$, SERGIO MUÑOZ ${ }^{2, b}$, \\ MARCELA LEGÜE C. ${ }^{1}$, LUIS BUSTOS ${ }^{2, c}$, MAURICIO BARRÍA P. ${ }^{3, d}$
}

\section{Psychomotor development in offspring of mothers with post partum depression}

Background: Postpartum depression (PPD) has adverse effects on psychomotor development of the offspring. Aim: To evaluate the relationship between PPD and psychomotor development in children aged 18 months, consulting in primary care. Material and Methods: Cross-sectional study with 360 infants and their mothers. Children had their psychomotor evaluation at 18 months and mothers completed the Edinburgh Postnatal Depression Scale at 4 and 12 weeks postpartum. The prevalence of both PPD and psychomotor alteration was estimated. The association between $P P D$ and psychomotor alteration, including confounding variables, was estimated through logistic multiple regression analysis. Results: The prevalence of PPD and psychomotor alteration was 29 and 16\%, respectively. Mothers with PPD had twice the probability of having an offspring with psychomotor alteration (Odds ratio $=2.0$, confidence intervals $=1.07-3.68$ ). This probability was significantly higher among single mothers or those with an unstable partner. Conclusions: PPD has a detrimental impact on psychomotor development of children.

(Rev Med Chile 2013; 141: 464-470).

Key words: Child; Depression, postpartum; Psychomotor disorders, developmental.
L a depresión posparto (DPP) es una patología que se inicia dentro de las cuatro semanas siguientes al parto $^{1}$. Su prevalencia en Chile es de 10 a $36,7 \% \%^{2,5}$, pero algunas cifras internacionales señalan de 5 a $18 \%{ }^{6,7}$. La tasa de detección en nuestro país sigue siendo baja, y sólo una cuarta parte de las pacientes recibe atención y tratamiento oportuno ${ }^{8,9,10}$. Síntomas habituales del puerperio tales como, cansancio, trastornos del sueño, inapetencia, falta de energía y baja de la libido, son similares a los de una depresión, hecho que enmascararía el problema y en consecuencia haría que las madres no consulten por este motivo ${ }^{3}$.

Existe amplia evidencia del impacto adverso que la DPP produce en el desarrollo infantil. Algunos estudios señalan que la falta de apego o patrones de interacción negativos madre-hijo, du- rante la etapa temprana de formación de vínculos, impactan el desarrollo futuro del niño, aun cuando la depresión de la madre haya finalizado ${ }^{11-13}$.

Se considera que la DPP es un factor de riesgo que influye negativamente en el desarrollo cognitivo y emocional del niño ${ }^{14}$. Por ejemplo, en los recién nacidos de madres deprimidas existiría mayor reactividad fisiológica (aumento de frecuencia cardiaca y niveles de cortisol), lo que se traduce en mayor irritabilidad y menor tolerancia al estrés ${ }^{11,15-17}$. Asimismo, en lactantes se ha reportado menor actividad general, afecto plano, y cambios electroencefalográficos similares a los que ocurren en adultos con depresión crónica ${ }^{11,18}$; además, a los 18 meses estos niños presentan peor desempeño en la escala de evaluación de desarrollo psicomotor de Bayley ${ }^{19}$. 
Se señala que el impacto de DPP continúa en edades avanzadas de los niños; por ejemplo, en preescolares existiría mayor frecuencia de trastornos de conducta, ansiedad, agresividad, retraso del lenguaje, y bajo desarrollo socio-emocional ${ }^{20,21}$; también se ha observado mayor dificultad de adaptación y concentración en escolares, abuso de sustancias, psicopatologías y agresividad en edades mayores ${ }^{22-24}$.

Se ha relacionado a la depresión materna con un mayor riesgo de maltrato infantil, más necesidad de servicios de educación especial por problemas de aprendizaje, mayores tasas de accidentes domésticos, y finalmente un aumento del riesgo de alteraciones conductuales en los hijos ${ }^{25-27}$.

Por otra parte, la aplicación de instrumentos específicos para evaluar DSM en niños menores de 2 años ${ }^{28,29}$, ha permitido que en nuestro país se pueda identificar a la población infantil en riesgo, y a la vez implementar programas de estimulación de su desarrollo. Algunos estudios señalan una prevalencia de alteración del DSM de aproximadamente $10,5 \%$ a los 18 meses de edad, y de 6,6\% a los 4 años ${ }^{15,30}$.

En la comuna de Valdivia, lugar donde se desarrolla este estudio, se producen aproximadamente 2.000 partos anuales ${ }^{5}$; si se extrapolan las cifras de prevalencia de DPP nacional, se esperaría que 200 a 700 de estas madres presenten DPP.

En este contexto, los controles habituales de salud del niño en los servicios de Atención Primaria, al menos durante los dos primeros años de vida, constituyen una oportunidad para pesquisar tanto la DPP de la madre como la alteración del DSM de sus hijos.

El objetivo principal de este estudio ha sido estimar la asociación entre la presencia de DPP de las madres y la alteración del DSM de sus hijos a los 18 meses de edad, en control en el sistema público de Atención Primaria de la comuna de Valdivia.

\section{Material y Método}

Estudio observacional de corte transversal, que incluyó al total de la dupla madre-hijo, que asistió durante el año 2006 al control de los 18 meses de edad, en los cuatro centros de atención primaria de salud (APS) de la ciudad de Valdivia-Chile. En este control normalmente se aplica a los niños la Escala de Evaluación del Desarrollo Psicomotor de los 18 meses de edad. Desde la ficha clínica se obtuvo el puntaje obtenido por sus madres en la Escala de Depresión Posnatal de Edimburgo $(\mathrm{EDPE})^{31}$; la que había sido aplicada entre las $4 \mathrm{y}$ 12 semanas posterior al parto.

Los criterios de inclusión de las madres fueron: haber completado la EDPE con puntaje consignado en la ficha, firmar consentimiento informado, y ser usuaria del sistema público de atención primaria de la ciudad de Valdivia. Se consideraron como criterios de exclusión maternos, no saber leer y escribir, y ser portadora de enfermedad psiquiátrica consignada en la ficha del lactante.

Los criterios de exclusión del niño fueron ser recién nacido de muy bajo peso de nacimiento, lactante portador de alguna genopatía, tener antecedentes de asfixia severa o Apgar a los 5 min menor a 6 , y ser segundo gemelo.

\section{Instrumentos}

\section{Escala de depresión posnatal de Edimburgo y} datos maternos

La escala se aplicó a las madres de todos los niños nacidos entre el 1 de enero y 31 de diciembre del año 2006 que se controlaban en el sistema de APS de la ciudad de Valdivia-Chile. La EDPE ha sido validada en diversos países, y en Chile por Jadresic et $\mathrm{al}^{32,33}$. Consiste en un instrumento autoaplicado de 10 ítems de selección múltiple; cada ítem tiene cuatro posibles respuestas ( 0 a 3 ). Arroja una puntuación de 0 a 30 y cualquier puntaje igual o superior a 10 indica presencia de DPP.

Por otra parte, en la ficha clínica de las madres se recolectaron datos sociodemográficos tales como: edad al parto, estado civil dividido en tres categorías: casada, soltera, conviviente; nivel de escolaridad estratificado en mayor o menor a 12 años de estudio. No se incluyó evaluación socioeconómica de las madres ya que todas ellas eran beneficiarias del sistema público de salud, lo cual ya las ubica en un rango determinado para efectos del estudio.

\section{Escala evaluación desarrollo psicomotor y datos biosociodemográficos en niños}

El DSM fue evaluado a los 18 meses mediante la escala de evaluación del DSM ${ }^{34}$. Esta evaluación arroja los siguientes cocientes: normalidad $(0,85-$ $2,0)$, riesgo $(0,70-0,84)$ y retraso $(\leq 0,69)$.

Los antecedentes clínicos considerados relevantes en el desarrollo del niño -según literatura 
científica-, fueron obtenidos desde su ficha clínica; entre ellos se consideró: peso de nacimiento, Apgar a los $5 \mathrm{~min}$, score o puntaje de riesgo de infección respiratoria alta (IRA), malnutrición por exceso, riesgo de desnutrición, antecedentes de riesgo de DSM, y lactancia materna exclusiva (LME) al $6^{\circ}$ mes.

\section{Análisis estadístico}

Los datos fueron ingresados en una planilla Excel y posteriormente procesados en el programa STATA 11.1. En una primera etapa se realizó análisis descriptivo exploratorio de todas las variables, para lo cual se utilizaron medidas de resumen (medias, desviaciones estándar, y rangos), distribuciones de frecuencia y tablas de asociación. En la estimación de prevalencia utilizamos intervalos de confianza de $95 \%$, y en la evaluación de asociaciones una significación de $5 \%$.

En primer lugar se determinó la prevalencia de DPP en las madres; considerando deprimidas a las mujeres con puntajes iguales o mayores de 10 en la EDPE. Se calculó la prevalencia de alteración del DSM (riesgo o retraso) en los hijos de estas madres según resultados de la evaluación del DSM a los 18 meses de edad. Se consideró como DSM alterado a aquellos con cociente de desarrollo menor a 0,84 ; puntaje definido como el punto de corte.

En una segunda etapa se realizó análisis estratificado de la relación DPP y DSM, controlando por variables biosociodemográficas de la dupla madre-hijo y antecedentes clínicos del niño.

El estudio fue aprobado por el Comité de Ética científico de la Universidad Austral de Chile, se obtuvo autorización de los centros de salud correspondientes, y se solicitó el consentimiento informado de las madres.

\section{Resultados}

La población de estudio estuvo constituida por 360 niños y sus madres. La edad promedio de las madres fue de 25 años (DS =7,2), 77\% de ellas tenía menos de 12 años de estudio; y más de la mitad era soltera o conviviente (Tabla 1).

Los niños fueron 180 hombres y 180 mujeres, el peso promedio al nacer fue de $3.410 \mathrm{~g}$, y $96 \%$ de ellos obtuvo un Apgar 5' de 9 puntos. Respecto de sus antecedentes clínicos considerados relevantes, se obtuvo que: a los 6 meses el $49 \%$ de los niños había presentado un score IRA leve-moderado,
$75 \%$ no tenía antecedentes de riesgo o retraso de DSM en controles anteriores, $91,1 \%$ no presentaba riesgo de desnutrición, ni tampoco de malnutrición por exceso (55\%); y 58\% mostraba LME a los 6 meses (Tabla 2).

En relación a la medición de la DPP, los resultados arrojaron que $29 \%$ de las madres obtuvo un puntaje igual o superior a 10 en la EDPE, con una media de 7,1 (DS = 5,5, rango 0-25). La evaluación de DSM de sus hijos a los 18 meses arrojó una prevalencia de $13,3 \%$ de niños en riesgo y $3,1 \%$ con retraso; es decir, el 16,4\% de los niños presentaba DSM alterado.

Al evaluar la asociación entre DPP de la madre y DSM alterado en los niños, los datos demostraron que la probabilidad que una madre con DPP tuviera un hijo con DSM alterado fue dos veces más que en aquella que no tenía $\mathrm{DPP}(\mathrm{OR}=2,0$, IC $=1,07-3,68)$.

La Tabla 3 muestra el efecto de la DPP sobre DSM estratificado por las características biodemográficas de la dupla madre-hijo, y algunos antecedentes clínicos de los niños. Se demostró que el efecto de la DPP en la alteración del DSM a los 18 meses fue independiente de la edad $(\mathrm{p}=0,70)$ y escolaridad $(\mathrm{p}=0,11)$ de la madre. Pero, la DPP en las madres con más de 12 años de estudio era 6 veces más que en las madres de menos estudio $(\mathrm{OR}=6,0, \mathrm{IC}=0,97-32,5)$. Respecto del estado civil se observa que la situación de convivencia modificó significativamente la relación DPP y DSM

Tabla 1. Características sociobiodemográficas de las madres

\begin{tabular}{|lcc|}
\hline Variable & $\mathbf{n}$ & $\%$ \\
\hline Edad & & \\
$13-19$ & 91 & 25,3 \\
$20-29$ & 216 & 47,8 \\
$30-45$ & 97 & 26,9 \\
Edad promedio & 25,3 & \\
DS & 7,2 & \\
Rango & {$[13-45]$} & \\
Escolaridad & & \\
$<=11$ años & 280 & 77,8 \\
$>=12$ años & 80 & 22,2 \\
Rango & {$[2,5-9,6]$} & \\
Estado civil & & \\
Soltera & 143 & 39,7 \\
Conviviente & 102 & 28,3 \\
Casada & 115 & 32 \\
\hline
\end{tabular}


Tabla 2. Características biodemográficas y clínicas de los niños

\begin{tabular}{|c|c|c|}
\hline Variable & $\mathbf{n}$ & $\%$ \\
\hline \multicolumn{3}{|l|}{ Peso al nacer } \\
\hline $1.500-2.000$ & 20 & 5,6 \\
\hline $2.500-3.500$ & 189 & 52,5 \\
\hline $3.500 y+$ & 151 & 41,9 \\
\hline Peso promedio & 3.410 & \\
\hline DS & 516 & \\
\hline Rango & [1.650-5.200] & \\
\hline \multicolumn{3}{|l|}{ Apgar 5' } \\
\hline $6-7-8$ & 14 & 3,9 \\
\hline $9 y+$ & 346 & 96,1 \\
\hline \multicolumn{3}{|l|}{ Sexo } \\
\hline Femenino & 180 & 50 \\
\hline Masculino & 180 & 50 \\
\hline \multicolumn{3}{|c|}{ Antecedentes clínicos } \\
\hline \multicolumn{3}{|l|}{ Score IRA (6 ms) } \\
\hline Leve & 209 & 58,1 \\
\hline Moderado & 112 & 31,1 \\
\hline Severo & 39 & 10,8 \\
\hline \multicolumn{3}{|l|}{ Riesgo retraso DSM } \\
\hline Sí & 90 & 25 \\
\hline No & 270 & 75 \\
\hline \multicolumn{3}{|l|}{ Riesgo desnutrición } \\
\hline Sí & 32 & 8,9 \\
\hline No & 328 & 91,1 \\
\hline \multicolumn{3}{|c|}{ Malnutrición por exceso } \\
\hline Sí & 162 & 45 \\
\hline No & 198 & 55 \\
\hline \multicolumn{3}{|l|}{ LME } \\
\hline 6 meses $y+$ & 210 & 58,3 \\
\hline Menos 6 meses & 150 & 41,7 \\
\hline
\end{tabular}

alterado a un nivel de $\mathrm{OR}=4,7, \mathrm{IC} 1,30-17,83, \mathrm{p}=$ 0,02 ; en este mismo contexto observamos que la relación DPP y DSM alterado fue 3,2 veces más frecuente en las madres solteras.

En un nivel de análisis más complejo podemos decir que la influencia de la DPP en la alteración del DSM fue independiente $(\mathrm{p}>0,25)$ de las características de los niños: sexo, bajo peso de nacimiento, antecedentes de mal nutrición por exceso, score de IRA, LME menor a seis meses, y antecedentes de riesgo de desnutrición o de retraso del DSM. Sin embargo, se observa que el efecto de DPP en el DSM alterado fue casi cinco veces mayor en niños que habían tenido IRA grave (OR
Tabla 3. Efecto de DPP sobre alteración de DSM según variables sociodemográficas y clínicas

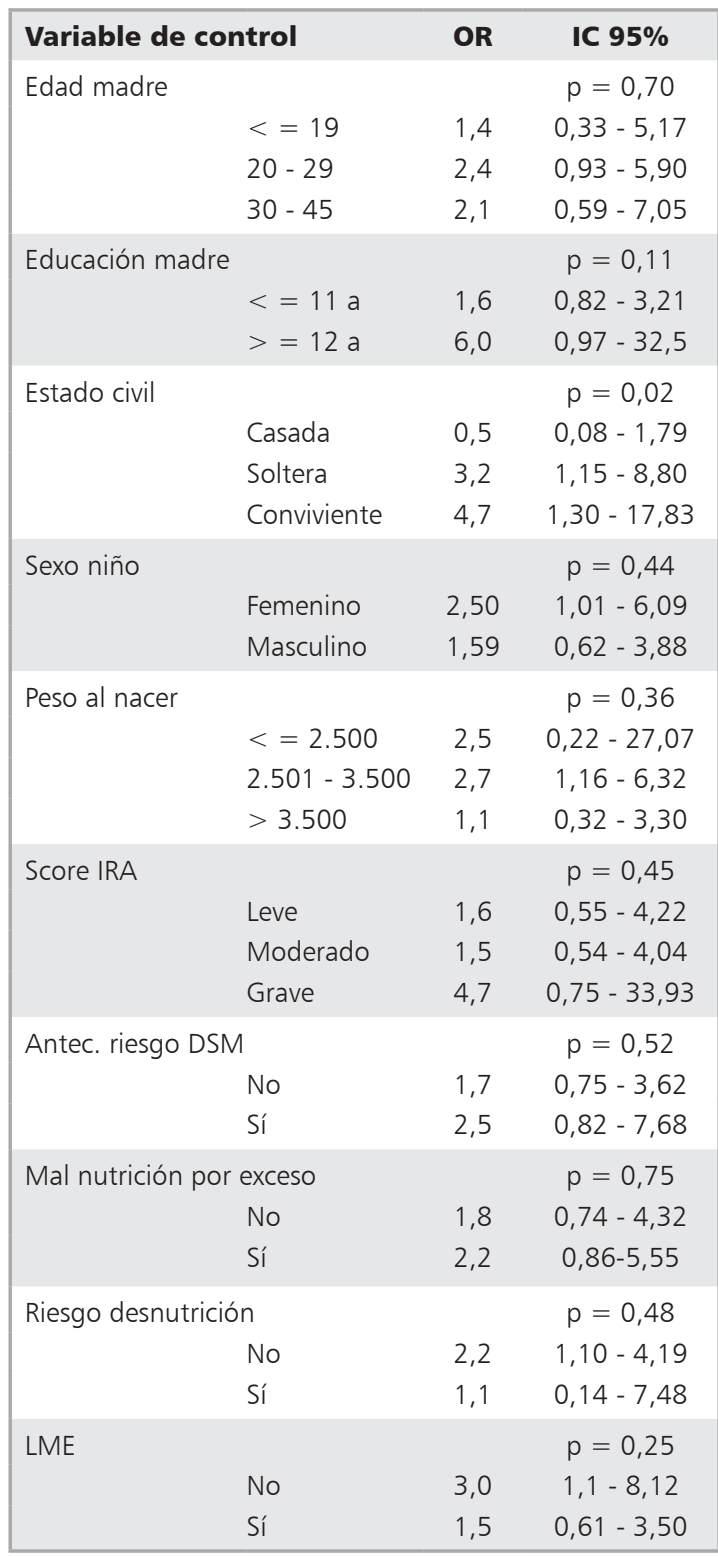

$=4,7, \mathrm{IC}=0,75-33,93), \mathrm{y}$ tres veces en niños que tenían una LME menor a seis meses $(\mathrm{OR}=3,0$, $\mathrm{IC}=1,1-8,12)$.

\section{Discusión}

Abordar los factores que amenazan o condicionan el desarrollo armónico del niño implica 
focalizarse en fenómenos biológicos, estructurales y psicosociales, entre ellos la DPP. Su importancia como factor asociado al desarrollo infantil ha sido ampliamente reconocida ${ }^{24,27}$. Los estudios que alertan de su alta prevalencia a nivel mundial, enfatizan que los efectos deletéreos pueden ser permanentes y trascendentes no sólo en la madre, sino también en la salud y desarrollo psicoemocional de su hijo, en consecuencia tanto su pesquisa como tratamiento debe ser oportuna ${ }^{35-38}$.

En Chile, la incorporación de la Escala de Depresión Posnatal de Edimburgo en el sistema público de APS, tiene gran importancia epidemiológica y clínica. Por una parte, permite generar evidencia sobre su prevalencia, riesgo y factores asociados; y por otra, permite aumentar la pesquisa de las madres deprimidas en el nivel primario. Esto otorgaría una oportunidad de tratamiento específico a madres que no tienen habitualmente acceso a otro tipo de atención.

Este estudio encontró una prevalencia de 29\% de DPP, cifra muy cercana a las más altas descritas en la literatura. Esto indica que casi un tercio de las madres que acude a un servicio público con sus hijos de año y medio tiene depresión, este hecho debería alertar al personal de salud en la búsqueda de mecanismos de pesquisa temprana, y de tratamiento oportuno en APS. Por ejemplo, Austin ${ }^{39}$ señala que la pesquisa de variables de riesgo en las madres durante las primeras consultas pediátricas y su consecuente incorporación a grupos de autoayuda, contribuirían a mejorar en forma importante el vínculo madre-niño y la calidad de vida de la madre lactante.

Este estudio descubrió que las madres solteras, con vínculos de pareja inestable, o madres con mayor escolaridad; presentaron mayor prevalencia de DPP; es decir -aun con las limitaciones propias del diseño-, podríamos aventurar que estas variables constituyen factores de riesgo importantes de observar en la práctica clínica.

Sin duda el resultado más relevante del estudio ha sido proporcionar evidencia de una asociación entre DPP de la madre y el DSM alterado, por ejemplo, se constata que una madre con DPP presenta dos veces más riesgo a que su hijo tenga alguna alteración del DSM $(\mathrm{OR}=2,0, \mathrm{IC}=1,07$ $3,68)$. Este hallazgo es muy congruente con el desarrollo teórico sobre el tema, y se demuestra en una población muy asequible para el equipo de salud de Atención Primaria en Chile.
Se observó además que esta relación (DPP y DSM alterado) es independiente de la edad de la madre $(\mathrm{p}=0,70)$ y de su escolaridad $(\mathrm{p}=0,11)$.

No obstante, surgió una asociación que amerita ser profundizada en futuros estudios; y es que a "mayor escolaridad de la madre, mayor DPP y en consecuencia mayor DSM alterado de sus hijos". También se observa que la situación de vínculo de pareja inestable modifica la relación DPP y DSM alterado; hay casi cinco veces más posibilidad que una madre con DPP y vínculo inestable tenga un hijo con DSM alterado.

Este estudio muestra una prevalencia de 16\% de alteración en el DSM en niños de 18 meses de edad, cifra concordante con algunos estudios a nivel nacional ${ }^{15,30}$. Se observa además, que variables clínicas consideradas importantes para el desarrollo del niño, como por ejemplo: Apgar a los $5 \mathrm{~min}$, estado nutricional, presencia de IRA, LME hasta los seis meses, son independientes de la alteración en su DSM ( $\mathrm{p}>0,25)$.

Sin embargo, el efecto de la DPP en el DSM alterado, fue mayor en niños que habían presentado una historia de IRA grave ( $\mathrm{OR}=4,7, \mathrm{IC}=0,75$ 33,93), o su LME había sido inferior a seis meses $(\mathrm{OR}=3,0, \mathrm{IC}=1,1-8,12)$.

Este trabajo puede considerarse pionero en estimar la asociación entre la DPP y DSM en niños chilenos e indagar acerca de los factores asociados a su riesgo. No obstante, la identificación precisa de estos factores, será un desafío para diseños de investigación más complejos.

En síntesis, los hallazgos indican: existencia de una relación entre DPP de la madre y DSM alterado en el hijo; mayor frecuencia de DPP en una madre soltera o con pareja inestable, y mayor frecuencia de DPP en una madre con más de 12 años de estudio. Asimismo, es importante como equipo de salud estar alerta en presencia de niños con antecedentes de LME menor a 6 meses, o que hayan presentado episodios graves de IRA; condiciones que se mostrarían gravitantes en la relación DPP y alteración del DSM.

\section{Referencias}

1. American Psychiatric Association. Diagnostic and Statistical Manual of Mental Disorders, 4th ed. Washington DC; 1994.

2. Consejo Asesor Presidencial para la Reforma de las Políticas de Infancia. El Futuro de los niños es siempre 
hoy. Documento Propuestas junio de 2006. Disponible en: www.crececontigo.cl/wp-content/uploads/2009/12/ El-Futuro-de-los-Ninos-es-Siempre-Hoy-Propuestasdel-Consejo-Asesor-Presidencial-para-la-Reforma-delas-Pol\%C3\%ADticas-de-Infancia.pdf [Acceso 20 de junio de 2011].

3. Jadresic E, Araya R. Prevalencia de la depresión posparto y factores asociados en Santiago de Chile. Rev Med Chile 1995; 123: 694-9.

4. Evans M, Vicuña M, Marín R. Depresión posparto, realidad en el sistema público de atención de salud. Rev Chil Obstet Ginecol 2003; 6: 491-4.

5. Servicio de Salud Valdivia. Estadísticas. Disponible en: www.ssvaldivia.cl-estadísticas/indicadores/partos [Acceso 18 de enero de 2010].

6. Misri S, Lussin S. Depression in pregnant women: clinical features and consequences. Disponible en: http:// www.uptodate.com/index [Acceso 25 de junio de 2009].

7. Lussin S, Misri S. Postpartum blues and depression. Up to Date, Feb. 2007.

8. Rojas G, Fritsch R, Solis J, Jadresic E, Castillo C, González $\mathrm{M}$ et al. Treatment of postnatal depression in low income others in primary-care clinics in Santiago, Chile: a randomised controlled trial. Lancet 2007; 370: 1629 37.

9. Jadresic E, Nguyen DN, Halbreich U. What does Chilean research tell us about postpartum depression? J Affect Disord 2007; 102: 237-43.

10. Postnatal Depression and Puerperal Psychosis. A National Clinical Guideline. Scottish Intercollegiate Guideline Network-National Government Agency. Disponible en: www.sign.ac.uk/guidelines/fulltext/60/index.html [Acceso 20 de abril de 2009].

11. Field T. Infant of depressed mothers. Infant Behaviour \& Development 1995; 18: 1-13.

12. Lussikin S, Pundiak T, Habib S. Perinatal depression: hiding in plain sight. Can J Psychiatry 2007; 52 (8): 47988 .

13. Jadresic E, Araya R, Jara C. Validation of the Edinburgh postnatal depression scale (EDPS) in Chilean postpartum women. Psychosom Obstet Gynecol 1995; 16 (4): 187-91.

14. Josefsson Ann. Postpartum depression: epidemiological and biological aspects. Medical Dissertation No 781, Linkoping University, 2003.

15. Schonhaut L, Rojas P, Kaempffer R. Factores de riesgos asociados a déficit del desarrollo psicomotor en preescolares de nivel socioeconómico bajo: Comuna urbano rural, Región Metropolitana. Rev Chil Pediatr 2005; 76 (6): 589-98.

16. Forman D, O'Hara M, Stuart S, Gorman L. Effective treatment for postpartum depression is not sufficient to improve the developing mother-child relationship. Dev Psychol 2007; 19 (2): 585-602.

17. Moehler E, Brunner R, Wiebel A, Reck C, Resch F. Maternal depressive symptoms in the postnatal period are associated with long-term impairment of mother-child bonding. Arch Women's Ment Health 2006; 9 (5): 273-8.

18. Weinberg K, Tronick E. Emotional Care of the At-Risk Infant: Emotional Characteristics of Infants Associated with Maternal Depression and Anxiety. Pediatrics 1998; 102: 1298-304.

19. Righetti-Veltema M, Bousquet A, Manzano J. Impact of postpartum depression symptoms on mother and her 18-month old infant. Eur Child Adolesc Psychiatry 2003; 12: $75-83$.

20. Murray L, Hipwell A, Hopper A. The cognitive development of five-tear-old children of postnatally depressed mothers. J Child Psychol Psychiatry 1996; 37: 925-7.

21. Murray L, Sinclair D, Cooper P. The socioemotional development of five-yoar-Old cpildren of Postnatally depressed mothers. J Child Psychol Psychiatry 1999; 40: 1259-71.

22. Hay DF, Pawlby S, Sharp D, Asten P, Mills A, Kumar R. Intellectual problems shown by 11 year old children whose mothers had postnatal depression. J Child Psychol Psychiatry 2001; 42: 871-89.

23. Currie L, Rademacher R. The paediatrician role in recognizing and intervening in postpartum depression. Pediatr Clin N Am 2004; 51: 785-801.

24. Rojas G, Fritsch R, Guajardo V, Rojas F, Barroilhet S, Jadresic E. Caracterización de madres deprimidas en el posparto. Rev Med Chile 2010; 138: 536-42.

25. Hay DF, Pawlby S, Angold A, Harold GT, Sharp D. Pathways to violence in children of mothers who were depressed postpartum. Dev Psychol 2003; 39 (6): 1083 94.

26. Fritsch R, Montt ME, Solís J, Pilowsky D, Rojas MG. ¿Cómo es la salud mental de los hijos de madres deprimidas consultantes a servicios de atención primaria?. Rev Med Chile 2007; 135: 602-12.

27. Dale F. Hay, Susan Pawlby, Adrian Angold, Gordon H, Sharp D. Pathways to violence in children of mothers who were depressed postpartum. Dev Psychol 2003; 39 (6): 1083-94.

28. O'Hara MW, Swain AM. Rates and risk of postpartum depression: a Meta-analysis. Int Rev Psychiatry 1996; 8: 37-54.

29. Bedregal P, Breinbauer C. El desarrollo de un modelo para la evaluación de rezagos del desarrollo infantil en Chile. Rev Med Chile 2007; 135: 403-5.

30. Rodríguez S, Arancibia V, Undurraga C. Escala de eva- 
luación del desarrollo psicomotor para niños de 0 a 2 años. Santiago: Editorial Galdoc 1976.

31. Schonhaut L, Schonstedt M, Alvarez J, Salinas P, Armijo I. Desarrollo psicomotor en niños de nivel socioeconómico medio-Alto. Rev Chil Pediatr 2010; 81 (2): 123-8.

32. Cox JL, Holden JM, Sagovsky R. Detection of postnatal depression. Development of the 10-item Edinburgh Postnatal Depression Scale. Br J Psychiatry 1987; 150: 782-6.

33. Jadresic E, Jara C, Miranda M, Arrau B, Araya R. Trastornos emocionales en el embarazo y el puerperio: estudio prospectivo de 108 Mujeres. Rev Chil Neuro-Psiquiat 1992; 30: 99-106.

34. Rodríguez S, Arancibia V, Undurraga C. Escala de evaluación del desarrollo psicomotor para niños de 0 a 24 meses. Editorial Galdoc, Santiago de Chile, 1985.

35. Alvarado R, Rojas M, Monardes J. Cuadros depresivos en el posparto en una cohorte de embarazadas: construcción de un modelo causal. Rev Chil Neuro-Psiquiat 2000; 38 (2): 84-93.

36. Shakespeare Judy. Evaluation of screening for postnatal depression NSC. National Screening Committee, Institute for Health Sciences, Oxford, 2001.

37. Cuijpers P, Bränmark JG, Van Stratten A. Psychological treatment of postpartum depression: a meta-analysis. J Clin Psychol 2008; 64 (1): 103-18.

38. Interventions for Postpartum Depression Guideline. Registered Nurses Association of Ontario-Professional Association Disponible en: http://www.rao.org/Page. asp?PageID $=924 \&$ Content $I D=806$ [Acceso 20 de enero de 2009].

39. Austin MP. Targeted group antenatal prevention of postnatal depression: a review. Acta Pschiatr Scand 2003; 107 (4): 244-55. 